\title{
The intercultural trust paradigm: Studying joint cultural interaction and social exchange in real time over the Internet
}

\author{
Chisato Takahashi ${ }^{a}$, Toshio Yamagishi ${ }^{a}$, James H. Liu ${ }^{\text {b,* }}$, \\ Feixue Wang ${ }^{\mathrm{c}}$, Yicheng Lin ${ }^{\mathrm{d}}$, Szihsien Yu ${ }^{\mathrm{d}}$ \\ ${ }^{\text {a } H o k k a i d o ~ U n i v e r s i t y, ~ J a p a n ~}$ \\ ${ }^{\mathrm{b}}$ Centre for Applied Cross Cultural Research, School of Psychology, PO Box 600, \\ Victoria University of Wellington, Wellington, New Zealand \\ ${ }^{\mathrm{c}}$ Sun Yat-Sen University, China \\ ${ }^{\mathrm{d}}$ National Taiwan University, Taiwan
}

\begin{abstract}
Distance transcending technology of the Internet generated a new experimental paradigm for the study of intercultural, or joint cultural interaction between members of different societies. University students from Japan, China, and Taiwan participated in experiments involving participants from their own society and another society in real time using an intercultural trust paradigm derived from a game theoretic and evolutionary approach to social exchange. The modified trust game improves on the Prisoner's Dilemma Game by eliminating greed as an explanation for lack of cooperation: the truster unilaterally decides whether or not to trust their exchange partner, and the allocator then decides whether or not to divide the reward fairly between the two of them. Participants earned real money by playing six rounds of one-shot trust games with three in-group members and three out-group members over the Internet. Across three experiments involving two interacting societies each, Japanese were found to be less trusting and trustworthy exchange partners compared to cultural Chinese. This suggests that Japanese collectivism is based more on long-term assurance networks, whereas Chinese collectivism provides a more expansive, guanxi-based approach to building new social networks. Japanese also showed less in-group favoritism in both trust and trustworthiness (or conditional fairness) at the national-level compared to cultural Chinese, suggesting that culture-specific content (e.g., collective guilt for WWII) may influence ethnocentrism at the national level.
\end{abstract}

(C) 2007 Elsevier Ltd. All rights reserved.

Keywords: Trust game; Social exchange; Internet; Reciprocity; Chinese; Japanese; Taiwanese; Trustworthiness; In-group favoritism; Ethnocentrism; Fairness

A cultural revolution has been taking place in psychology ( $\mathrm{Ng} \& \mathrm{Liu}, 2000)$. Fuelled by globalization, immigration, and the rapid economic and intellectual development of Asia, Latin America, and the Middle East, cross-cultural psychology is becoming indispensable to a global psychology of the 21st century (Liu \& Ng, 2007; Yang, 2000). The "global village" is now an economic reality, with world trade reaching unprecedented levels, but world poverty is also endemic (Stiglitz, 2006). However, the methods employed by cross-cultural psychologists to achieve recognition from mainstream psychology have focused on limited aspects of the theoretical and practical knowledge necessary for a global psychological science. The strategy of cross-cultural comparison, where results of experiments and surveys

\footnotetext{
* Corresponding author.

E-mail address: James.Liu@vuw.ac.nz (J.H. Liu).
} 
from one society are compared and most often contrasted with that from another society or societies strikes at the heart of mainstream assumptions about the universality of basic psychological processes (see the debate about the universality or cultural contingency of self-enhancement, e.g. Brown \& Kobayashi, 2003; Heine, 2003). But it does not do enough to advance theoretical knowledge about how people from different cultures cooperate with one another, perhaps the more central question in globalization.

Current methods of cross-cultural comparison are in some sense autistic because the participants from the two or more societies studied never interact with one another. Only in the area of acculturation (Ward, Furnham, \& Bochner, 2001) do participants in research actually report on their experiences with people from other cultures; this work is almost exclusively survey-based, and involves one culture as the host society and potentially assimilative rather than treating two societies or cultures equally. Hence, there is a need for a new method of cross-cultural investigation that we introduce as an "intercultural" paradigm or "joint cultural" experiment (Mashima, Yamagishi, \& Macy, 2004). We use the distance transcending technology of the Internet (see Bakir, Landis, \& Noguchi, 2004) coupled with a game theory approach to social exchange (Yamagishi, 1998) that allows us to assess interpersonal, group, and intergroup aspects of intercultural encounters. Intercultural experiments are conducted in real time, within the comfort and control of computer laboratories separated by thousands of miles, affording both engagement and control in a single experimental paradigm.

One of the most interesting theoretical issues of the age of globalization is how people from different cultures are able to trust one another enough to engage in fair or reciprocal social exchange. As Cook et al. (2005) point out, there is always risk involved in establishing any cooperative relationship, and trust may emerge as the outcome rather than the cause of cooperation. The experimental study of behavior in dyadic exchanges involving a forced choice between two alternatives is among the most widely used paradigms to study social exchange in the social and economic sciences (Kagel \& Roth, 1995; Kollock, 1998). Because the outcomes in social exchange can be represented numerically, translated into dollar amounts and rewarded accordingly, they are more informative than attitudes or values as analogues for economic behavior.

To psychologists, the most familiar among the "social dilemmas" used to model exchange is the prisoner's dilemma game (PDG), where the reward structure gives each partner a motivation to cheat the other, but if both defect they receive the least desirable joint outcome. The problem with the PDG is that its reward structure conflates greed with lack of trust; a player in the PDG may defect because he or she wants to exploit their partner to obtain the maximum reward, or because he or she is afraid of being exploited by the other player (i.e., lacks trust). Using variations on the theme of the PDG, Yamagishi, Kanazawa, Mashima, and Terai (2005) demonstrated the importance of separating trust from cooperation. In this paper we introduce another variation on the theme of social dilemmas through a "Trust Game" that allows us to separate greed from trust in participants' choices for social exchange. This paradigm gives clear and separate measures of willingness to trust the exchange partner, to reciprocate trust, and to treat the partner fairly.

Like the PDG, the trust game (Kreps, 1990) takes the form of a social exchange between players, with mixed motives to cooperate or defect. However, the trust game disentangles some of the various motivations for cooperative behavior bundled together in the PDG game. The truster in this situation must decide unilaterally whether or not to trust their partner by giving them the opportunity to divide either a large amount of money between them (the trust option), or a small amount (not trusting), and the partner decides to allocate either fairly (share equally) or unfairly (take all). Hence, the measure of trust derived from this situation is entirely based on prior expectancies about what the partner will do (or altruism), uncontaminated by any motivation to exploit the partner. Unlike in the standard trust game, we modified the role of allocator to give them a chance to be fair in dividing a smaller amount of reward even when they were not trusted. This allows us to derive measures of conditional and unconditional fairness on the part of the allocator (depending on whether the truster has decided to give them a larger or smaller amount to divide between them).

Our modified trust game has the advantage of being able to provide measures of both trust and fairness in intercultural transactions. The first player (the truster) must unilaterally decide whether or not to entrust the second player with a large reward to divide between the two of them; the second player (the allocator) decides whether to divide the reward equally (fairly) or to take the lion's share. From an evolutionary perspective (Yamagishi, 2007), the strongest pressure to cooperate (i.e., reciprocate) exists for an allocator when an in-group member has trusted them. This type of cooperation, which we call "conditional fairness" or "trustworthiness", is the type of in-group favoring pact necessary and evolutionarily adaptive for small-group survival. In-group members should feel morally obliged to reciprocate trust with other clearly identified in-group members; violating this rule invites punishment from other 
in-group members (Boehm, 1999). To cooperate with an in-group member when they have trusted you is also to expect to be treated in the same benevolent manner yourself: this type of behavioral system deals with free-riders and makes reciprocal altruism sustainable (Yamagishi \& Kiyonari, 2000).

Yamagishi, Jin, \& Kiyonari (1999) demonstrated that this type of in-group favoritism was found for Japanese in minimal groups, as long as both partners were mutually aware of one another's group membership. By extending their results to the national level, we can examine whether intercultural behavior deviates significantly from behavior in minimal groups having no cultural baggage. If we fail to find in-group favoritism in conditional fairness among Japanese in our modified version of the trust game, this would suggest that there are national-level group norms that inhibit the default operation of a group-based "social exchange heuristic", where reciprocal cooperation is expected as a "ticket" for entry into a generalized system of trustful exchange among in-group members.

\section{Beyond the minimal group: social identity and cross-cultural differences}

Cross-cultural psychology to date has focused on using dimensions of cultural variation such as individualism/ collectivism or power distance (Hofstede, 1980; Smith \& Bond, 1999) to predict differences in attitudes or behavior depending on the nature of self-construal in a given culture (e.g., Markus \& Kitayama, 1991). Far less emphasis has been placed on issues of social identity and intergroup relations where members of different cultures must interact with one another to achieve (or fail to achieve) desirable outcomes (Hilton \& Liu, in press). The theory of independent and interdependent selves (Markus \& Kitayama, 1991) circumvents the literature on social identity and intergroup relations by claiming that tendencies towards individual (independent) versus collective/group-oriented (interdependent) behavior are a function of culture-based self-construal.

The model of self-provided by the social identity/self-categorization theory is more flexible: it claims that any member of any culture may at times act independently, as an individual following personal attitudes or beliefs, and at other times interdependently, according to group norms (Turner, Hogg, Oakes, Reicher, \& Wetherell, 1987). According to self-categorization theory, social comparison based on the makeup of people and goals present will determine the sense of self that becomes salient in a situation (Turner et al., 1987; Reicher \& Hopkins, 2001), which will in turn determine resource transactions. The intercultural trust paradigm described here is ideally suited for the study of intergroup relations between cultures or societies, and opens a paradigmatic portal for the study of how groups and group membership influence trust and fair play in situations involving joint behavior between members of different societies. The measures of reciprocity and fair play derived from the allocator require this exchange partner to integrate individuating information about their partner (i.e., did they trust me or not?) with categorical features like ingroup versus out-group status.

The rise of a social identity-based approach to intergroup relations has to a significant extent relied on the "minimal group paradigm" (Tajfel, Billig, Bundy, \& Flament, 1971) where strangers are brought into the lab, and in the absence of any interaction between them, a new social identity or self-categorization is created through a perceptual judgment task. False feedback is given to participants that their judgments on the task indicate that they belong to different social categories (or groups) like "Klees versus Kandinskys" or "dot over-estimators versus dot under-estimators" where in fact assignment to groups is random. This "minimal group identity" has been shown to produce in-group favoritism (see Hewstone, Rubin, \& Willis, 2002 for a recent review). The ability to create intergroup behavior in the laboratory, and to create and manipulate social identities with no prior history or beliefs has spawned an explosion of publications and theoretical interest. However, Tajfel (Israel \& Tajfel, 1972) initially thought of the minimal group situation as an opportunity not to explore "empty" social identities, but as a first step for the progressive examination of how group norms function as culture-based mediators of behavior in intergroup situations.

Following Leyens, Yzerbyt, \& Schadron (1994), Yamagishi et al., 2005a) suggested that the minimal group paradigm functions as a "maximal group paradigm" in the sense that there are no group-based cues for behavior in the situation except the group label. Counter-intuitively, they showed that in-group favoritism among Japanese is found in a minimal group situation (Yamagishi et al., 1999), but when Japanese believed they were interacting with other Japanese and Australians. Both Australians and Japanese displayed out-group favoring tendencies when they believed they were interacting with one another (but actually deception was involved) in a PDG (Yamagishi et al., 2005). The group norms and stereotypes operant in the situation were such that Japanese thought Australians were more honest/ trustworthy than their own nationality, and Australians wanted to show that they were fair and unprejudiced in dealing with Japanese. These data suggest that ethnocentrism and in-group favoritism is to a significant extent a cultural 
phenomenon, and governed by content-rich information like group norms, auto- and hetero-stereotypes, and prior histories of group interaction (Liu \& Hilton, 2005; Hilton \& Liu, in press).

\section{Interactive forms of collectivism within the dimensions of cultural variation}

Yamagishi et al.'s (2005) results hence suggest that ethnocentrism and in-group favoritism may be interactive phenomena, a product of joint cultural interaction rather than unilateral cultural tendencies such as individualism or collectivism. Their prediction (hypothesis 3, p. 178) that Japanese, as collectivists who share the belief that ingroup favoring behavior is common among group members should be more cooperative with in-group members compared to Australians was not confirmed. In fact both "individualistic" Australians and "collectivist" Japanese increased in-group cooperation when they knew their interaction partners were aware of their nationality compared to when their partners were not aware of the commonality of national group membership, but not to the point where in-group cooperation significantly exceeded cooperation with the out group. There was no real intercultural interaction in this experiment, as participants were deceived into believing they were interacting with people from another culture. The present research extends these findings to real intercultural interaction via the Internet.

Yamagishi and colleagues (Yamagishi, 1988; Yamagishi \& Yamagishi, 1994; Yamagishi, Cook, \& Watabe, 1998) have long argued that Japanese are low on general trust compared to Americans; they prefer to rely on assurance networks of established relationships where cheating on your partner would be bad for your reputation and sanctioned by other members of the social network. Hence, the overall level of cooperation among Japanese in experimental dilemmas of social exchange involving strangers has been shown to be lower than among participants of individualistic cultures (e.g., Yamagishi \& Yamagishi, 1994). Yamagishi et al. (2005) observed that "the high level of mutual cooperation frequently observed in a collectivist culture, in particular in Japanese culture, is a product of collectivist social institutions that monitor and control individual members' behavior. . . Thus, in a group artificially created in the laboratory where no formal or informal institutions monitor and sanction individuals' behavior, participants from a collectivist culture tend to expect a lower level of cooperation from others and they themselves cooperate at a lower level" (p. 186).

The issue addressed in the present study is whether these tendencies observed among Japanese collectivists can also be generalized to Chinese in interactions involving the two cultures. While Japanese and Chinese are used interchangeably in the cross-cultural literature as exemplars of collectivist cultures, many of the social institutions of assurance described by Yamagishi and Yamagishi (1994) would appear to be weaker among urban and educated Chinese speaking populations. Japan is an island nation, and the only place in the world where most people speak Japanese. Social relations have been stable and well established during a long period of prosperity following recovery from World War II. China, on the other hand, covers a huge territory, where the label "Chinese language" covers a plethora of mutually unintelligible "dialects". Social relations in China have been in upheaval not only with the recent liberalization of the economy, but throughout an extended history of 170 years involving the collapse of the Qing dynasty, foreign invasion, and internal calamity. Fukuyama (1995) argued that Chinese trust is restricted to family and kin more than Japanese, and hence China constitutes a lower-trust society compared to Japan.

On the other hand Chinese people have been more adventurous than Japanese in establishing minority Chinese speaking enclaves all over the world, where strangers who are ethnically Chinese must establish new social relations with in-group members, building up networks of "guanxi" (Hwang, 1998; Chou, Cheng, Huang, \& Cheng, 2006) rather than relying on established assurance networks. Hence, among Chinese cooperation may well be mediated by a more expansive orientation towards building up in-group trust even in the absence of pre-existing assurance mechanisms. From a cultural psychology perspective, in contrast to Fukuyama (1995) we might expect a higher level of trust and more in-group favoritism in reciprocating trust among Chinese compared to Japanese in social exchange involving strangers, based on an expansive form of collectivism reliant on building up trustworthy relations with ingroup members. In Japan, a more defensive form of collectivism involving trust and trustworthy behavior only in wellestablished social relations may be more prevalent. Finally, Taiwanese are culturally Chinese but in recent years have come to see themselves as a political entity distinct from the mainland (Huang, Liu, \& Chang, 2004). Hence, the inclusion of Taiwan allows us to observe both culture and society level effects by comparing Chinese and Taiwanese behavior. 
In summary, we focus on culture and society-level tendencies in trust, reciprocity, and fair play among Japanese, Chinese, and Taiwanese in intercultural interaction with one another. Our first goal is to describe properties of a modified trust game that provides separate measures of trust, reciprocity, and fairness as intercultural phenomena.

In terms of specific hypotheses, we examined culture-specific manifestations of intercultural behavior in three East Asian societies all characterized as collectivist and high power distance. First, based on findings from the minimal group paradigm and social identity theory, we predicted a main effect for in-group status: that is, all societies should favor the in-group more than the out group in terms of trust and conditional fairness/trustworthiness (Brewer, 1979). Second, we predicted lower levels of trust and trustworthiness on the part of Japanese because collectivism in Japan is more a function of established relations in assurance networks, whereas among cultural Chinese it is more of a fluid process of guanxi or network building (these are society-level main effects). Third, we made a soft prediction of no interaction between participant society and in-group status on any of the dependent variables, following both the evolutionary arguments developed earlier, and social identity theory. All societies should equally trust and reciprocate trust among in-group members unless there is compelling group-level, or normative pressure to behave otherwise (Israel \& Tajfel, 1972). This is a soft hypothesis because both our previous experimental data (Yamagishi et al., 2005) and theories of history and identity (Liu \& Hilton, 2005) contradict this universality hypothesis. Fourth, we predicted no differences between societies or groups on unconditional fairness, because non-trusters have already violated the implicit contract of in-group favoring behavior expected among members of a collective; unconditional fairness comes close to altruism, which is probably better characterized as an individual difference rather than a reliable group-based difference.

\section{Methods}

We conducted three experiments where participants from three different societies participated via Internet. We compared Japanese, Chinese and Taiwanese on their willingness to trust and to reciprocate trust with other participants from their own society and another society. Two laboratories in two different societies were connected via Internet during any one experiment. The three experiments involved Japanese-Chinese, Japanese-Taiwanese, and ChineseTaiwanese. Results are analysed at a society-level, comparing Japan versus Mainland China and Taiwan.

\subsection{Participants}

Participants were undergraduate students in the three respective societies. In the Japan-China experiment 132 participants from each society participated (including one Japanese confederate). In the Japan-Taiwan experiment there were 104 participants from each society. In China-Taiwan experiment there were 108 participants from each society (including one Taiwanese confederate). Confederates were required because each session required four participants from each country; if one participant did not show up for a session we filled the position with a confederate. We excluded the confederates and two Taiwanese participants in Japan-Taiwan experiment and three Taiwanese participants in China-Taiwan experiment from our analysis because they suspected the monetary reward aspects of the experimental situation very strongly. Each session consisted of four participants from each society, a total of eight per session. In all sessions, care was taken to ensure that the participants did not know one another and had no chance to interact with one another except via the experiment.

The participants from Japan were 124 men and 112 women from Hokkaido University (average age = 19.1). The participants from Taiwan were 88 men and 124 women (average age 21.0). Most were from National Taiwan University (70\%) and others from nearby universities around Taipei. The participants from China were 120 men and 120 women (average age 19.9) from Sun-Yat-Sen University in Southwestern China (Guangzhou). The main incentive for participation in all societies was monetary reward.

\subsection{Apparatus}

The experiments were conducting on PCs with Windows. Display screens were designed using Visual Basic 6.0 software, and Internet communication was managed through a modified version of winsock control. An Internet connection was used for the Japan-Taiwan experiment, whereas a dial up connection was used in experiments involving China. 


\subsection{Trust game}

The trust game was played by participants who did not have any opportunity for face-to-face interaction before, during or after the experiment. Participants played six rounds of a one-shot trust game with another participant (either an in-group or an out-group member). In each session two of the participants from each society were randomly assigned the role of truster and the other two were assigned the role of allocator. Participants repeated this trust game six times with a different partner each time. Each participant was paired with an in-group member three times, and with an out-group member three times in a random order.

The participant who is assigned the role of truster makes a binary choice between a High Trust and Low Trust option. When a truster chooses the Low Trust option, both truster and allocator receive 90 Points (P) each, and the allocator is given an opportunity to divide a common share of $60 \mathrm{P}$ between the two partners. When the truster chooses the High trust option, both truster and allocator receive 30P each, and the allocator is given an opportunity to divide a common share of 300P between them. The participant who is assigned the role of allocator makes a binary choice between Take All and Share Equally. When an allocator chooses to Take All, they get all the common points and the truster does not get any. When an allocator chooses Share Equally, both allocator and truster get the half of common points.

If a truster chooses High Trust and the allocator chooses Share Equally, both receive 180P (150P (common points) in addition to 30P (fixed points)). If a truster chooses Low Trust and the allocator chooses Share Equally, both receive 120P (30P (common points) in addition to 90P (fixed points)). If a truster chooses High Trust and the allocator chooses Take All, the truster receive only 30P (fixed points), while the allocator receive 330P (300P (common points) in addition to 30P (fixed points)). If a truster chooses Low Trust and the allocator chooses Take All, the truster receive 90P (fixed points) while the allocator receive 150P (90P (common points) in addition to 60P (fixed points), see Fig. 1 for summary).

Our experimental variant on the standard trust game gives us three measures of cooperation with regards to the allocator's behavior (see Fig. 1). When faced with a Low Trust choice, a Share Equally decision by the allocator can be interpreted as unconditional fairness; that is, the allocator divides the common share equally despite a failure to offer the potential for greater reward by the truster. When faced with a High Trust choice, on the other hand, a Share Equally choice can be interpreted as conditional fairness (or trustworthiness) on the part of the Allocator. The difference between the two (proportion of Share Equally when faced with High Trust minus proportion of Share Equally choices when faced with Low Trust) provides a measure of the effect of being trusted (or reciprocity).

The unit of reward for the trust game in our computer program was called a "Point". At the end of the experiment, each point the participants accumulated during the experiment was converted into cash, and each participant was paid according to how many points they had earned during the game: $1 \mathrm{P}=1.5$ Yen in Japan, $0.02 \mathrm{RMB}$ in China, and 0.3 NTD in Taiwan. These amounts were calibrated according to the average amount it cost participants to have lunch at the cafeteria in their university. Japanese participants earned about 1,330 Yen with a minimum of 270 Yen and a maximum of 2,970 Yen. Chinese participants earned about 17.67 RMB with a minimum of 6.0 RMB and a maximum

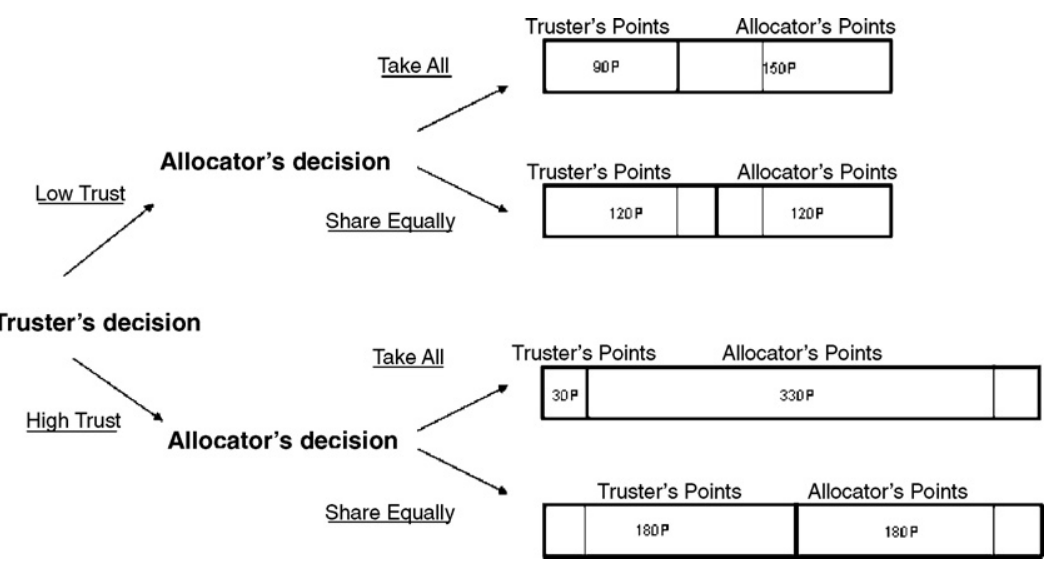

Fig. 1. Reward structure for the trust game. 
of 36.0 RMB. Taiwanese participants earned about 269 NTD with a minimum of 99 NTD and a maximum of 549 NTD. The amount earned was designed to be psychologically and functionally similar in each society based on purchasing power rather than a direct function of currency conversion. Participants were informed of the conversion rate for their own currency in their own society, but not that of participants from another society.

We used a 3 participants' society (Japan, China, Taiwan) $\times 2$ (group: in-group vs. out-group) factorial design with the former a between-subjects factor and the latter a within-subject factor. This description of the experimental design does not include the society of the out-group - for example, Chinese interacted with either Taiwanese or Japanese - as a factor. Only aggregated results of each society (ignoring the nationality of the out group) are provided here as we wanted to focus on methodological aspects of the paradigm and culture/society level findings rather than bilateral relations between two societies here (analyses of the three experiments treated separately will be provided in subsequent papers).

\subsection{Procedure}

Upon arrival at the laboratory, each participant was immediately led to a personal computer (PC) that had partition boards separating it from other computers so participants could not see or interact with one another in a face-to-face manner. Each participant was provided with an ID number by a receptionist upon arrival that was not known to the experimenter in the computer room who greeted them. After all participants arrived in each laboratory, the room experimenters gave the consent forms and asked participants to sign them. After collecting the consent forms, the experimenters set up the PC screens on each desk, and asked participants to enter their ID number, and sex. When all eight participants put in their IDs, the experiment started. This experiment was conducted by PCs including the instructions for the trust game.

One round of the trust game was conducted in the following way. (1) Both truster and allocator were given information about their partner's society of origin. (2) The truster made a decision between a Low Trust and a High Trust Choice, labelled as Choice X and Choice Y as shown in Fig. 1 (these choices were not labelled as Low or High Trust). While the truster was choosing, the allocator made a prediction about the truster's choice and awaited their decision. (3) The actual decision of the truster was communicated to the allocator via the Internet. (4) The allocator made their choice (Take All or Share Equally), labelled as Choice S and Choice T. While the allocator was choosing, the truster made a prediction about allocator's choice (not analysed here). Each participant had to wait for all others to finish their choices before the entire group could move on to the next round of the game.

Participants were given two trial runs to familiarize them with the game that did not count towards their point totals. Trusters were not given the allocator's decision after each trial; rather they were given feedback as to their point totals and allocator decisions after all trials were finished. This ensured that the experience of being fairly or unfairly treated by in-group and out-group partners would not affect Trusters' choices. An experimenter in the control room who was aware only of their ID number calculated the amount of money they earned in the game and gave this in an envelop for the room experimenter to deliver to the participants. In this way, the behavior of the participants was kept confidential from everyone, including the room experimenter.

After the participants finished all their trials, the experimenter gave them a post-experiment questionnaire. After participants filled out this questionnaire, they left the laboratory after being paid individually.

\subsection{Measures}

The three basic dependent variables derived from the trust game are trust, unconditional fairness, and conditional fairness. From these we can also derive measures of overall fairness (by combining unconditional and conditional fairness) and reciprocity (by subtracting unconditional from conditional fairness). Participants randomly assigned to the role of truster had only trust scores, while those assigned to the role of allocator had fairness and reciprocity scores.

\subsubsection{Trust}

Trust rate was calculated as the proportion of High Trust decisions by a truster. It is transformed from a binary response in each trial (High Trust vs. Low Trust) to a continuous variable by calculating the proportion of High Trust choices across the six rounds of the game where points were being accumulated. In addition to overall trust, we constructed measures of trust for in-group partners and trust for out-group partners. As mentioned previously, these 
measures separate expectancies for cooperative behavior from the partner from personal motivations for greed, and hence should be sensitive to such group-level factors as stereotypes or norms. Further, lack of feedback about Allocator's behavior kept the Truster's experiences during the experiment from influencing his or her choices.

\subsubsection{Unconditional fairness}

Unconditional fairness was the proportion of Share Equally decisions made by an allocator when faced with a Low Trust decision by the trusters across the six rounds. It is transformed from a binary response in each trial (Take All vs. Share Equally) to a continuous variable by summing Share Equally decisions and dividing by the number of Low Trust choices faced. Allocators who never faced a Low Trust decision on any of the six rounds did not receive an unconditional fairness score (about 2-3 participants in each society). In addition to overall unconditional fairness, we constructed unconditional fairness scores for in-group partners and for out-group partners. Unconditional fairness is the decision we expect to be least influenced by in-group/out-group factors because the implicit social pact "favoring the in-group" has already been violated by the Low Trust decision on the part of the Truster.

\subsubsection{Conditional fairness (or trustworthiness)}

Conditional fairness or trustworthiness was the proportion of Share Equally decisions made by an allocator when faced with High Trust decisions from trusters across the six rounds, following the same procedures described for unconditional fairness. This is the decision wherein we expect the most in-group favoritism in the absence of societylevel norms or beliefs specifying otherwise.

\subsubsection{Overall fairness}

Overall fairness was the overall proportion of Share Equally decisions made by an allocator across all six rounds regardless of truster decisions. This is perhaps a less interesting measure because it conflates two theoretically distinct measures of fairness.

\subsubsection{Reciprocity}

Reciprocity was represented by the bias in the ratio of Share Equally choices made between when an allocator is trusted (a truster chooses High Trust) and when an allocator is not trusted (a truster chooses Low Trust). That is, it is conditional fairness minus unconditional fairness, with a positive score indicating a greater tendency to be fair when the partner is trusting, and a negative score indicating a greater tendency to be fair when the partner is not trusting. We constructed two measure of reciprocity in addition to the overall measure: with in-group partners and with out-group partners.

\section{Results}

All statistical analyses used the following format: first, data across the three experiments were aggregated; then, a $2 \times 2$ analysis of variance (ANOVA) was conducted on the focal dependent measure with participant society of origin and partner's national group (in vs. out). A main effect of group indicates differences between in-group versus outgroup members (predicted in hypothesis 1 for trust and trustworthiness). A main effect of participant society indicates society level differences between Japan, China, and Taiwan on the dependent measure (predicted for trust and trustworthiness in hypothesis 2). An interaction indicates that one society was more in-group favoring than other(s) (hypothesis 4 makes a soft prediction of no interactions for all dependent variables; hypothesis 3 predicts no main effects or interactions for unconditional fairness).

\subsection{Trusters' behavior}

\subsubsection{Trust}

Fig. 2 shows the average levels of Trust with in-group partners and with out-group partners across three societies. A participant society of origin $\times$ partner's group (same society or out-group) ANOVA shows that the main effect of partner's group $(F(1,337)=11.86, p<.01)$ was significant. In accord with our first hypothesis, participants trusted ingroup partners $(M=0.50$, S.D. $=0.35)$ more than out-group partners $(M=0.44$, S.D. $=0.34)$. In accord with hypothesis 2 , the main effect of society $(F(2,337)=3.15, p<.05)$ was significant as well. Japanese participants 


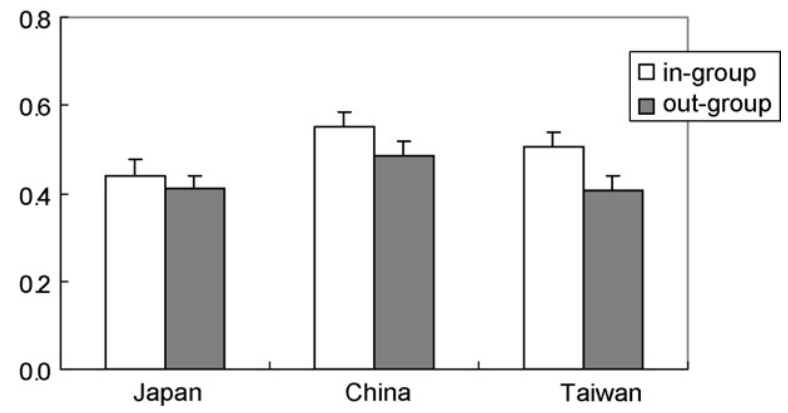

Fig. 2. Average level of trust for in-group and out-group partners by society of origin.

$(M=0.43$, S.D. $=0.31)$ were less trusting than Chinese $(M=0.52$, S.D. $=0.29)$ and Taiwanese $(M=0.46$, S.D. $=0.28)$. Both Chinese and Taiwanese trusted in-group partners more than out-group partners, whereas this ingroup trust was very weak among Japanese, but the society $\times$ partner's group interaction effect $(F(2,337)=1.08$, ns. was not significant (in accord with hypothesis 3).

\subsection{Allocators' behavior}

\subsubsection{Unconditional fairness}

Table 1 displays the average of unconditional fairness with in-group and out-group partners. A society $\times$ partner's group ANOVA shows that in accord with hypothesis 4, none of the main effects (society: $F(2,262)=2.10$, ns.; partner group: $F(1,262)=0.00$, ns.) or the interaction $(F(2,262)=0.12$, ns. $)$ were significant.

\subsubsection{Conditional fairness (or Trustworthiness)}

The most interesting results were obtained for conditional fairness, and somewhat counterintuitive. Fig. 3 displays the average of conditional fairness, or trustworthiness, with in-group partners and with out-group partners. A society $\times$ partner's group ANOVA shows that in accord with hypotheses 1 and 2, the main effect of partner's group $(F(1,224)=11.42, p<.01)$ and the main effect of society $(F(2,224)=3.38, p<.05)$ were significant. However, contradicting hypothesis 3 , the interaction effect of society $\times$ partner's group $(F(2,224)=4.95, p<.01)$, was also significant. Conditional fairness by the allocator when trusted by their partner was more pronounced when the partner was an in-group member compared to an out-group member among Chinese (with in-group, $M=0.68$, S.D. $=0.41$; with out group, $M=0.52$, S.D. $=0.44$ ) and Taiwanese allocators (with in-group, $M=0.59$, S.D. $=0.45$; with out group, $M=0.45$, S.D. $=0.44$ ). This pattern was not observed among the Japanese allocators (with in-group, $M=0.43$, S.D. $=0.46$; with out group, $M=0.49$, S.D. $=0.46$ ). Hence, in accord with predictions, both Chinese and Taiwanese were more trustworthy (i.e. divided the money equally) when the partner who trusted them was an in-group member

Table 1

Unconditional fairness for in-group and out-group partners as a function of society of origin

\begin{tabular}{|c|c|c|c|}
\hline \multicolumn{4}{|c|}{ Unconditional Fairness } \\
\hline & $n$ & $M$ & S.D. \\
\hline \multicolumn{4}{|l|}{ Japan } \\
\hline In-group & 104 & 0.41 & 0.43 \\
\hline Out-group & 106 & 0.39 & 0.43 \\
\hline \multicolumn{4}{|l|}{ China } \\
\hline In-group & 95 & 0.45 & 0.43 \\
\hline Out-group & 109 & 0.48 & 0.43 \\
\hline \multicolumn{4}{|l|}{ Taiwan } \\
\hline In-group & 90 & 0.52 & 0.44 \\
\hline Out-group & 95 & 0.49 & 0.46 \\
\hline
\end{tabular}




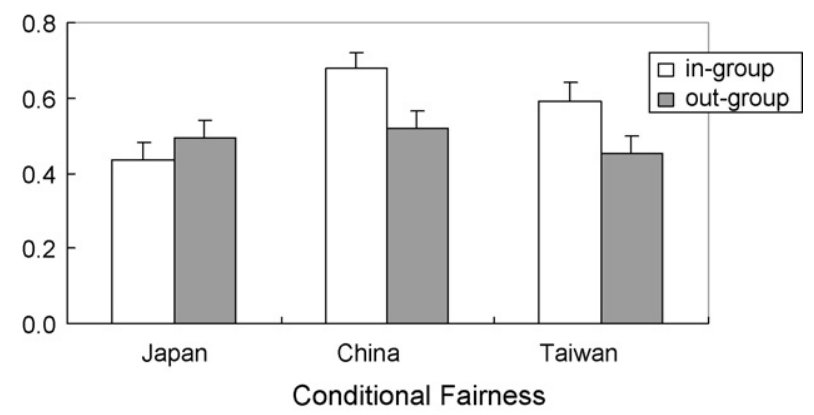

Fig. 3. Average level of conditional fairness or trustworthiness for in-group and out-group partners by society of origin.

compared to if they were from another society. Also in accord with hypothesis 2, Japanese allocators were also less trustworthy overall compared to cultural Chinese from both Taiwan and the mainland. Surprisingly, confounding hypothesis 3, there was a significant interaction between society and group status where Japanese allocators were not fairer when trusted by in-group members compared to out-group members, but cultural Chinese were; this suggests the presence of group-level information for Japanese participants to not act in a default, in-group favoring manner.

The three main dependent measures listed above can be further analysed as follows (but these results are less central to our main hypotheses).

\subsubsection{Overall fairness}

A society $\times$ partner's group ANOVA shows that the main effect of partner's group $(F(1,338)=10.56, p<.01)$, and the interaction effect of society $\times$ partner's group $(F(2,338)=3.86, p<.05)$ were significant. The main effect of society was marginal $(F(2,338)=3.01, p<.10)$. Both Chinese and Taiwanese were more fair (i.e. allocated equally) to in-group trusters more than out-group trusters (the average of the overall fairness among Chinese to in-group $=0.58$, S.D. $=0.32$, to out-group $=0.48$, S.D. $=0.35$; among Taiwanese to in-group $=0.57$, S.D. $=0.32$, to out-group $=0.48$, S.D. =0.36). This in-group favoring pattern was not observed among Japanese allocators (to in-group $=0.44$, S.D. $=0.35$, to out-group $=0.45$, S.D. $=0.36$ ). These results amplify the results on conditional fairness described previously.

\subsubsection{Reciprocity}

Table 2 displays the average level of reciprocity to in-group partners and out-group partners. A society $\times$ partner's group ANOVA shows that the main effect of partner's group was significant $(F(1,161)=7.68, p<.05)$. Overall, there was more reciprocity to in-group members than out-group members. The main effect of society was not significant $(F(2,161)=0.60$, ns. $)$. Chinese participants showed more reciprocity when they played with in-group partners $(M=0.23$, S.D. $=0.61)$ than with out-group partners $(M=0.01$, S.D. $=0.61)$. Japanese and Taiwanese participants

Table 2

Reciprocity for in-group and out-group partners as a function of society of origin

\begin{tabular}{lcrr}
\hline Reciprocity & & & \\
& $n$ & & \\
\\
\hline Japan & & & \\
$\quad$ In-group & 80 & 0.04 & 0.11 \\
$\quad$ Out-group & 84 & & 0.56 \\
China & 84 & 0.23 & 0.61 \\
$\quad$ In-group & 76 & 0.01 & 0.61 \\
Out-group & & & \\
Taiwan & 75 & 0.08 & 0.62 \\
$\quad$ In-group & 74 & -0.02 & 0.59 \\
$\quad$ Out-group & & & \\
\hline
\end{tabular}


showed little tendency to reciprocate more when partnered with an in-group member. But since the interaction between society $\times$ partner's group $(F(2,161)=1.81$, ns. $)$ was not significant, we should be cautious about focusing too much on these differences.

\section{Discussion}

Among the most interesting results of the intercultural trust paradigm concerned the society-level differences in ingroup favoritism, trust, and trustworthiness between Chinese, Japanese, and Taiwanese people. First, in accord with hypothesis 2, Japanese people displayed less in-group favoritism than either Chinese or Taiwanese on most measures. Providing partial support for hypothesis 1, Japanese displayed slight in-group favoring tendencies to trust, and among Chinese and Taiwanese this pattern was more pronounced (the interaction term did not reach significance, confirming hypothesis 3). Second, counterintuitively and against hypothesis 3, Japanese failed to show in-group favoritism in trustworthiness (conditional fairness) in sharp contrast to both Chinese and Taiwanese. Reciprocating in-group trust should have strong moral connotations for being a good in-group member, and being accepted as a participant in a generalized system of cooperative exchange among in-group members (Yamagishi, 2007). In our experiment, however, Japanese were not more trustworthy to one another than to Chinese in decisions involving real money.

As predicted in hypothesis 2, Chinese appeared more open to establishing new trustful relationships in our experimental situation compared to Japanese. This suggests that Chinese and Japanese forms of collectivism may differ in that Chinese have a more expansive form of collectivism that is conducive to building up new relationships compared to Japanese, who tend to be more trusting and trustworthy only in long-established assurance-based networks, where cheating is sanctioned by social circles. Results defy Fukuyama's (1995) argument that Japan is a high trust society compared to China, but rather emphasize among Chinese a "coalition building" approach rooted in a cultural psychology of guanxi (relationships, Chou et al., 2006). We suspect this expansive approach to trust may be more characteristic of Chinese elites on the Mainland and Taiwan rather than less educated members of the general Mainland Chinese population.

Outside of this surprising result concerning overall trust, the behavior of cultural Chinese (Mainlanders and Taiwanese) in the intercultural trust paradigm was less surprising. As expected from standard intergroup theory (hypothesis 1), they showed in-group favoritism on both trust and trustworthiness. Both social identity theory and the group heuristic predict in-group favoritism in a situation involving trust when behavior is based only on prior expectancies or altruism, and trustworthiness, where there is specific evidence of the partner's willingness to cooperate. According to the group heuristic (Yamagishi, 2007; Yamagishi \& Kiyonari, 2000), trustworthiness (or conditional fairness) is based on a default sense of reciprocal obligation to in-group members, and should occur when there is mutual knowledge of group membership and there is an absence of specific norms or beliefs specifying otherwise. Social identity theory provides several accounts of when to expect in-group favoritism (e.g., Grieve \& Hogg, 1999; Hogg \& Abrams, 1988; Hunter, 2005), but it is commonly associated with high status groups whose position is perceived as legitimate (Tajfel \& Turner, 1979) on dimensions of importance to the group.

As anticipated, in accord with hypothesis 4 there were no society-level or group-level effects on unconditional fairness. This variable seems to primarily reflect individual differences in altruism rather than operate as a function of group-level factors. In all three societies, there was no in-group favoritism in allocation when participants were not trusted. Hence, our measures of trust and conditional fairness (or trustworthiness) were shown to have a substantially different psychological meaning compared to our measure of unconditional fairness, which appears to be linked to more individual-level factors.

Consistent with a prior study that used deception to mimic the intercultural experimental paradigm (Yamagishi et al., 2005), we found that Japanese did not show in-group favoritism in trustworthiness in-group interactions involving nationality. In a prior study involving Japanese and Australians imagining they were in intercultural interactions, Japanese participants showed out-group rather than in-group favoritism. These results are surprising to social psychologists who expect in-group favoritism to be a general phenomenon (but see Heine, 2003), and to lay people who believe popular press reports about the ethnocentrism of Japanese, but they are consistent with results from the World Values Survey (2005). These data indicate that Japanese people are significantly less proud about their nationality compared to Chinese. That is why we termed hypothesis 3 a "soft hypothesis": it is based on prevailing assumptions in social psychology about the universality of in-group favoring behavior, but these theories do not take social context and history sufficiently into account. 
We suspect that the current results may be due to a particularly Japanese sense of national identity when faced with people from other East Asian societies that Japan invaded or occupied during and before the Second World War. Liu and Hilton (2005) have argued that history provides a major source of content for national identity, and that both Japanese and Germans face the unenviable position of having to atone for sins committed during WWII (see also Liu et al., 2005, for cross-cultural data indicating that WWII is the most important event in world history across 12 societies). Young people in Japan are schooled that nationalism in the form of in-group favoritism or out-group derogation is unacceptable, and part of the cause of the great suffering Japan people experienced as a consequence of their defeat in WWII. Similar patterns have been identified in post-war Germany (see for example Dresler-Hawke \& Liu, 2006). The current results suggest that far from being universal, ethnocentrism in real social categories such as nationality can be very much the product of culture-specific learning (see Hilton \& Liu, in press; Liu \& Laszlo, in press). In other words, culture specific content, like collective guilt (Branscombe \& Doosje, 2004), can over-ride the generalized tendency towards in-group favoritism found among minimal groups. The importance of this finding should not be under-estimated given the extent to which contemporary social psychological theory on intergroup relations depends on de-contexualized laboratory experiments involving fictional or relatively unimportant social categories. The findings are all the more striking because we paid participants meaningful amounts of money in these experiments, unlike the points or attitudes used as dependent measures in most social psychological studies.

As a final point of discussion, we would like to invite future research using intercultural experimental paradigms, like the trust game described here or the prisoner's dilemma used in Yamagishi et al., 2005. Joint cultural experiments have the advantage of assessing cultural differences involving real interaction between members of different societies separated by great distances. The trust game described here has particularly desirable properties for the investigation on intergroup relations. Through careful pilot testing, we calibrated the incentive structure in our game so that the behavioral decisions reported are almost exactly at 50\%. That is, about half the participants chose to trust their partner, and half did not; about half the participants choose to allocate fairly to their partner, and half did not. This would appear to be ideal for further experimental manipulations that increase or decrease the salience of group membership or increase or decrease the amount of conflict between groups. While the details will depend on the particular cultures involved, the calibration of average decisions at 50\% in the current study suggests that experimenters are unlikely to encounter floor or ceiling effects as they examine interactions between new societies or manipulate factors that serve to increase or decrease cooperation. Furthermore, the separate measures of trust, unconditional fairness, and trustworthiness are differentially sensitive to group and individual level factors.

The research reported in this paper represents our initial step toward finding ways to improve trust between people separated not only by a long distance but also by different cultures, and embedded in different social settings. The results of our study invite more questions than we can answer in this particular study. For example, are the low levels of trust and trustworthiness exhibited by Japanese participants in this study unique to the use of nationality as a group? Or, do they represent a more general tendency of low trust and low cooperativeness among the Japanese in situations involving a lack of institutional assurance? Results of cross-cultural studies of questionnaire survey and experimental games such as the PDG and other social dilemmas consistently indicate that Japanese tend to be low in trust and cooperativeness compared to Americans (Kiyonari \& Yamagishi, 1999; Yamagishi, 1988; Yamagishi et al., 1998; Yamagishi \& Yamagishi, 1994), Australians (Yamagishi et al., 2005), and New Zealanders (Yamagishi, Mifune, Liu, \& Pauling, 2007). There are some studies (Wang \& Yamagishi, 1999, 2005) that show that Japanese are low in trust compared to Chinese as well. Yamagishi (1998) argues that a lack of generalized trust is an important feature of a collectivist society, but it seems to be a defining feature of the Japanese form of collectivism. Liu and Atsumi (in press) by contrast point to specific features of "collective guilt" over negative behavior in World War II.

The Chinese form of collectivism, which is more network-based than group/category-based, requires a higher level of trust for expanding networks. A second question concerns the nature of trust and fairness by Taiwanese participants. Their pattern of trust and allocation generally falls in-between Chinese and Japanese, potentially implying the impact of modernization accompanied by general economic wealth, expanded middle class, and democratic political systems. These seem to be responsible for the weak level of in-group bias in trust and fairness, but no definite conclusions can be drawn from the current study.

Internet and distance transcending technologies allow social scientists to explore phenomena that could not be explored in previous generations. We hope that this paper will stimulate collaboration between laboratories in many parts of the world, and lead to new scientific discoveries about how to facilitate cooperation across national boundaries. 


\section{Acknowledgments}

The authors gratefully acknowledge the support of grant RG04-P-03 from the Chiang Ching-Kuo Foundation for International Scholarly Exchange, and support from the 21st Century COE "Cultural and Ecological Foundations of the Mind" at Hokkaido University that helped make this research possible.

\section{References}

Bakir, A., Landis, D., \& Noguchi, K. (2004). Looking into studies of heterogenous small groups. In D. Landis, J. M. Bennet, \& M. J. Bennett (Eds.), Handbook of intercultural training (3rd Ed., pp. 416-434). Thousand Oaks, CA: Sage.

Boehm, C. (1999). Hierarchy in the forest: The evolution of egalitarian behaviour. Cambridge, MA: Harvard University Press.

Branscombe, N., \& Doosje, B. (2004). Collective guilt. Cambridge: Cambridge University Press.

Brewer, M. B. (1979). In-group bias in the minimal intergroup situation: A cognitive-motivational analysis. Psychological Bulletin, 86, 307-324.

Brown, J. D., \& Kobayashi, C. (2003). Culture and the self-enhancement bias. Journal of Cross-Cultural Psychology, 34(5), $492-495$.

Chou, L. F., Cheng, B. S., Huang, M. P., \& Cheng, H. Y. (2006). Guanxi networks and members' effectiveness in Chinese work teams: Mediating effects of trust networks. Asian Journal of Social Psychology, 9(2), 79-95.

Cook, K., Yamagishi, T., Cheshire, C., Cooper, R., Matsuda, M., \& Mashima, R. (2005). Trust building via risk taking: A cross-societal experiment. Social Psychology Quarterly, 68(2), 121-142.

Dresler-Hawke, E., \& Liu, J. H. (2006). Collective shame and the positioning of German national identity. Psicologia Politica, 32, $131-153$.

Fukuyama, F. (1995). Trust: The social virtues and creation of prosperity. London: Penguin.

Grieve, P. G., \& Hogg, M. A. (1999). Subjective uncertainty and intergroup discrimination in the minimal group situation. Personality and Social Psychology Bulletin, 25(8), 926-940.

Heine, S. (2003). Making sense of East Asian self-enhancement. Journal of Cross-Cultural Psychology, 34(5), 596-602.

Hewstone, M., Rubin, M., \& Willis, H. (2002). Intergroup bias. Annual Review of Psychology, 53(1), 575-604.

Hilton, D.J., \& Liu, J.H. (in press). Culture and inter-group relations. Social representations of history and historical charters. In R. Sorrentino \& S. Yamaguchi (Eds.), The Handbook of Motivation and Cognition: The Cultural Context (Vol. 3). New York: Guilford.

Hofstede, G. (1980). Culture's Consequences: International differences in work-related values. Beverly Hills, CA: Sage.

Hogg, M., \& Abrams, D. (1988). Comments on the motivational status of self-esteem in social identity and intergroup discrimination. European Journal of Social Psychology, 18(4), 317-334.

Huang, L. L., Liu, J. H., \& Chang, M. (2004). The "Double Identity" of Taiwanese Chinese: A dilemma of politics and culture rooted in history. Asian Journal of Social Psychology, 7(2), 149-189.

Hunter, J. A. (2005). Threats to group value, domain-specific self-esteem and intergroup discrimination amongst minimal and national groups. British Journal of Social Psychology, 44(3), 329-353.

Hwang, K. K. (1998). Guanxi and mientze: Conflict resolution in Chinese society. Intercultural Communication Studies, 3(1), 17-37.

Israel, J., \& Tajfel, H. (1972). The context of social psychology: A critical assessment. Oxford: Academic Press.

Kagel, J., \& Roth, A. (Eds.). (1995). Handbook of experimental economics. Princeton: Princeton University Press.

Kiyonari, T., \& Yamagishi, T. (1999). A comparative study of trust and trustworthiness using the game of enthronement. Japanese Journal of Social Psychology, 15, 100-109 (in Japanese).

Kreps, D. M. (1990). Cooperative culture and economic theory. In J. Alt \& K. Shepsle (Eds.), Perspectives on positive political economy (pp. 90143). Cambridge: Cambridge University Press.

Kollock, P. (1998). Social dilemmas: The anatomy of cooperation. Annual Review of Sociology, 24, 183-214.

Leyens, J.-P., Yzerbyt, V., \& Schadron, G. (1994). Stereotypes and social cognition. London: Sage.

Liu, J.H. \& Atsumi, T. (in press). Historical conflict and resolution between Japan and China: Developing and applying a narrative theory of history and identity. In T. Sugiman, K.J. Gergen, W. Wagner, \& Y. Yamada (Eds.), Meaning in action: Constructions, narratives, and representations.

Liu, J. H., \& Hilton, D. (2005). How the past weighs on the present: Social representations of history and their role in identity politics. British Journal of Social Psychology, 44, 537-556.

Liu, J. H., Goldstein-Hawes, R., Hilton, D. J., Huang, L. L., Gastardo-Conaco, C., Dresler-Hawke, E., Pittolo, F., Hong, Y. Y., Ward, C., Abraham, S., Kashima, Y., Kashima, E., Ohashi, M., Yuki, M., \& Hidaka, Y. (2005). Social representations of events and people in world history across twelve cultures. Journal of Cross-Cultural Psychology, 36(2), 171-191.

Liu, J. H., \& Ng, S. H. (2007). Connecting Asian in global perspective: Special issue on past contributions, current status, and future prospects of Asian social psychology. Asian Journal of Social Psychology, 10(1), 1-7.

Markus, H., \& Kitayama, S. (1991). Culture and self: Implications for cognition, emotion and motivation. Psychological Review, 98, 224-253.

Mashima, R., Yamagishi, T., \& Macy, M. (2004). Trust and cooperation: A comparison of ingroup preferences and trust behavior between American and Japanese students. The Japanese Journal of Psychology, 75(4), 308-315 (in Japanese).

Ng, S. H., \& Liu, J. H. (2000). Cultural revolution in psychology. Asian Journal of Social Psychology, 3(3), $289-293$.

Smith, P. B., \& Bond, M. H. (1999). Social Psychology Across Cultures (2nd Ed.). Boston: Allyn \& Bacon.

Stiglitz, J. (2006). Making globalization work. London: Allen Lane.

Tajfel, H., Billig, M., Bundy, R., \& Flament, C. (1971). Social categorization in intergroup behaviour. European Journal of Social Psychology, 1, $149-178$. 
Tajfel, H., \& Turner, J. C. (1979). The social identity theory of intergroup behaviour. In S. Worchel \& W. Austin (Eds.), Psychology of intergroup relations (pp. 33-48). Chicago, IL: Nelson-Hall.

Turner, J. C., Hogg, M. A., Oakes, P. J., Reicher, S. D., \& Wetherell, M. S. (1987). Rediscovering the social group: A self-categorization theory. New York: Basil Blackwell.

Wang, F. X., \& Yamagishi, T. (2005). Group-based trust and gender-difference in China. Asian Journal of Social Psychology, 8(2), 199-210.

Wang, F. X., \& Yamagishi, T. (1999). Changing roles of trust in Chinese society. In James, H. L iu \& Colleen, Wa rd Eds. Progress in Asian Social Psychology. vol. 2 (pp.367-386). Seoul, Korea: Kyoyook-Kwahak-Sa.

Ward, C., Bochner, S., \& Furnham, A. (2001). The psychology of culture shock (2nd Ed.). London: Routledge.

World Values Study Group (2005). World Values Survey, 1999-2004. Ann Arbor, MI: Inter-university Consortium for Political and Social Research (ICSPR). http://www.worldvaluessurvey.org/.

Yamagishi, T. (1988). The provision of a sanctioning system in the United States and Japan. Social Psychology Quarterly, $51,32-42$.

Yamagishi, T. (1998). Trust: The evolutionary game of mind and society. Tokyo: Tokyo University Press.

Yamagishi, T. (2007). The social exchange heuristic: A psychological mechanism that makes a system of generalized exchange self-sustaining. In M. H. B. Radford, S. Ohnuma, \& T. Yamagishi (Eds.), Cultural and ecological foundations of the mind: Mutual construction of the mind and society. Sapporo, Japan: University of Hokkaido Press.

Yamagishi, T., Cook, K. S., \& Watabe, M. (1998). Uncertainty, trust, and commitment formation in the United States and Japan. American Journal of Sociology, 104, 165-194.

Yamagishi, T., Jin, N., \& Kiyonari, T. (1999). Bounded generalized reciprocity: Ingroup boasting and ingroup favoritism. Advances in Group Processes, 16, 161-197.

Yamagishi, T., Makimura, Y., Foddy, M., Matsuda, M., Kiyonari, T., \& Platow, M. J. (2005a). Comparisons of Australians and Japanese on groupbased cooperation. Asian Journal of Social Psychology, 8, 173-190.

Yamagishi, T., Kanazawa, S., Mashima, R., \& Terai, S. (2005b). Separating trust from cooperation in a dynamic relationship. Rationality and Society, 17(3), 275-308.

Yamagishi, T., \& Kiyonari, T. (2000). The group as the container of generalized reciprocity. Social Psychology Quarterly, 63, 116-132.

Yamagishi, T., \& Yamagishi, M. (1994). Trust and commitment in the United States and Japan. Motivation and Emotion, 18, $129-166$.

Yamagishi, T., Mifune, N., Liu, J. H., \& Pauling, J. (2007). Exchanges of group-based favors: Ingroup bias in the prisoner's dilemma game with minimal groups in Japan and New Zealand. Asian Journal of Social Psychology, in review.

Yang, K. S. (2000). Monocultural and cross-cultural indigenous approaches: The royal road to the development of a balanced global psychology. Asian Journal of Social Psychology, 3(3), 241-264. 